\title{
Effect of Daily Safety Briefing Huddles on the Reporting of Adverse Events and Near-misses
}

\author{
Minping Deng ${ }^{1}$, Weiju Chen ${ }^{2,}$, Tianying Pang ${ }^{1}$, Chunmei Lin ${ }^{1}$ \\ ${ }^{1}$ Gastrointestinal Surgical Unit, The First Affiliated Hospital of Jinan University, Guangzhou, China \\ ${ }^{2}$ Department of Nursing, The First Affiliated Hospital of Jinan University, Guangzhou, China \\ Email address: \\ dengminping@126.com (Minping Deng),weijuchen2008@163.com (Weiju Chen) \\ ${ }^{*}$ Corresponding author
}

To cite this article:

Minping Deng, Weiju Chen, Tianying Pang, Chunmei Lin. Effect of Daily Safety Briefing Huddles on the Reporting of Adverse Events and Near-misses. American Journal of Nursing Science. Vol. 8, No. 3, 2019, pp. 92-96. doi: 10.11648/j.ajns.20190803.12

Received: January 28, 2019; Accepted: March 14, 2019; Published: April 3, 2019

\begin{abstract}
Background: Incident reporting offers valuable information regarding safety issues, but near-misses (NM) and adverse events (AE) remain underreported. DSB huddles help foster collective situational awareness that increases an organization's capacity to respond to safety concerns. However, effects of DSB huddles on AE/NM reporting remain understudied. Objective: To examine how daily safety briefing (DSB) huddles operate in a surgical unit, and assess their impact on reporting of adverse events and near-misses. Methods: DSB huddles were piloted in a gastrointestinal surgical unit. The study compared AE/NM reporting rates and reporting types before and after adopting DSB huddles. Results: After adopting DSB huddles, AE reporting improved from $0.9 \%$ to $1.8 \%$, and NM reporting improved from $0.5 \%$ to $7.1 \%(\mathrm{p}<.05)$. Self-reporting of safety issues increased from $44.4 \%$ to $73.8 \%$; NM reporting domains increased from 6 to 15 . Conclusions: DSB huddles increased reporting rates of AE and of NM particularly, improved reporting dimensions of NM, and increased team members' situational patient safety awareness.
\end{abstract}

Keywords: Daily Safety Huddles, Incident Reporting, Near-misses, Risk Management, Patient Safety

\section{Introduction}

Patient safety is recognized as a key indicator of quality of medical care by most global health organizations and authorities, as well as by many researchers. In recent decades, medical errors and undesirable events have continued to increase globally, and present one of the most significant global challenges to modern health care, causing huge economic and societal burdens [1]. Safety concerns are broadly defined as "patient safety events that reached the patient, regardless of whether harm occurred; near-misses or close calls, which are patient safety events that did not reach the patient [2]. Adverse events (AE), which are safety events that reach the patient. AE and NM need to be identified, reported, and analysed effectively, and lessons learned need to be translated into practice and systems improvements. Patient safety reporting systems (PSRS) to identify and mitigate risks to patients who are harmed by medical care have become a priority and challenge to health care delivery over the last decades [1]. The implementation of PSRS allows analysis of accumulated data and their use for continuous improvement of medical care quality via feedback and learned lessons. With the development and implementation of PSRS, there has been some improvement in the disclosure and documentation of medical errors, but the vast majority remain unreported [3], because incident reporting by PSRS only captures events that have already occurred and has limited value in more real-time identification of safety concerns. Studies have demonstrated that individuals may be less likely to report an event when it does not cause patient harm $[4,5]$. Few studies have estimated the rate of near-misses, accurately identifying and analysing $\mathrm{NM}$ is more difficult. Unlike AE, which may be retrospectively identified through review of hospital records, NM may not be documented and may not be evident by review of medical records [6]. Previous studies have relied on survey results and incident reporting systems to estimate NM [5, 7]. Thus, there is a compelling need to increase awareness of these NM/'hidden' safety problems and to develop new, proactive strategies to identify safety concerns. Safety huddles 
could potentially be useful for learning proactively about safety concerns [8]. Safety huddles have been found useful in creating a sense of collective situational awareness that increases an organization's capacity to respond to safety concerns [8]. Safety huddles were designed to share information, review events, and help teams develop action plans for coordinated patient care [9]. Daily safety briefings (DSBs) are reports from front-line units and ancillary departments to enhance awareness of immediate or potential problems affecting patient safety and care, and enhance communication across all disciplines [10]. DSBs promote hospital-wide transparency and patient safety [11]. DSBs are usually employed hospital-wide [10,11], and few studies have studied DSB huddles conducted in the unit and their impact on the reporting of adverse events and NM. This study conducted at a single unit in the First Affiliated Hospital of Jinan University, Guangzhou, China. The idea was inspired by the U.S. Mid-Michigan Medical Center (Midland's: Daily briefings help improve safety culture).

\section{Methods}

\subsection{Setting and Participants}

The First Affiliated Hospital of Jinan University, Guangzhou, southern China, is a Grade-A Tertiary Hospital with a total of approximately 1800 beds, which integrates medical services, education, and a research hospital. DSB huddles took place in a 46-bed gastrointestinal surgical unit which provides care to approximately 1900 patients annually. The participants included 19 registered nurses (RNs), all of them in full-time positions.

At our institution, an electronic AE reporting system was developed to encourage a culture of safety and address barriers to reporting of AE. 14 special AE reporting templates related to the 14 basic nursing quality indexes of clinical nursing in Guangdong Province. One generic template for nurses to report other AE. Hospital policy is for all nursing AE to be reported through the electronic reporting system by individual nurses, but $\mathrm{NM}$ are not reported. The reports quality review is done by a nurse manager, who categorizes the reports and sends them electronically to risk management groups. Risk management groups analyse the AE every 3 three months, and problems were brought back to the nursing management department. There is no formal feedback system to individuals, and the unit nursing staff are not involved in the process. Meanwhile there is no formal system encouraging the reporting of NM.

The decision to explore implementation of DSB huddles was made by the nurse manager. The nurse manager was the project leader for this implementation. All nursing staff willingly participated in the project. The idea of implementing DSB huddles was approved by the nursing management team prior to planning. An internal review board reviewed this project and deemed it exempt from further human subjects review.

\subsection{Design}

This study was conducted as a before-after study. Data from 16 May 2016 to 16 May 2017 was pre-DSB group. DSB huddles were adopted from 10 June 2017 to 10 June 2018 as the post-DSB group. Demographic characteristics of participating nurses and patients were basically similar.

\subsection{Procedures}

\section{Phase I: Staff Education}

Before the start of the DSB huddles, a 2-hour educational in-service was developed and presented to nursing staff $(\mathrm{N}=$ 19). The education highlighted the definition of AE and NM, the importance of reporting, and the outcomes hypothesized from implementing DSB huddles. Nurse manager provided a verbal, step-by-step scenario to report patient safety issues, followed by a demonstration of the DSB huddles to the nursing staff.

\section{Phase II: Implementation}

The DSB huddles are a process by which registered nurses and nursing assistants come together daily for briefing about patient safety issues over the last 24 hours. The process is designed to identify and predict any concerns about aspects of patient safety. On 10 June 2017, the DSB huddles were initiated, and held in the treatment room immediately adjacent to the nurse station, to bring nursing staff together briefly from nearby locations. The meeting follows a standardized process so that it can be run by various people. The 'host' rotates between one nurse manager and four senior nurses. DSB huddles are brief, lasting between 5-15 minutes, and are often conducted standing. They were held at 3PM each afternoon, which was the beginning of the afternoon shift, and the timing of the nurse hand-over, and included the morning and afternoon shifts' nurses. The process is an in-person meeting, conducted in front of a white board, and all nursing staff on the day were to attend. The nurses going off-duty would report patient safety issues that had occurred in their areas, predictable issues in the next 24 hours in their area, and identify any safety issues that need to be discussed. In the DSB huddles, nurses would follow a customized reporting template: Safety/Quality Issues/Events within the last 24 hours, anticipated safety/quality/risk issues in the next 24 hours, any defects requiring follow up tracking. During the meeting, the nurse manager would call the nurses' names to report one-by-one in an orderly manner. If there were no safety problems to report, nurses simply answered 'safe' when the nurse manager called their name. If the nurse had a safety issue to report, in addition to reporting AE, safety risks, and predicted risks, it was also necessary for the nurse to state who or what departments helped to resolve the problem for further discussion and formulation of countermeasures after the meeting. If the raised safety issue had been solved independently within the unit, the moderator would lead the discussion at once with extra emphasis on who, what, and how they helped to solve the problem. For other safety issues that needed inter-department cooperation, the nurse manager would contact other department leaders to discuss the solution 
within 24 hours. The moderator would lead the discussion, record the data (recording all concerns discussed in the safety huddles) and write down the newly identified items on the whiteboard. After each DSB huddle, an electronic summary about safety and risk status was posted on a WECHAT GROUP to all nursing staff. This way, even those not in attendance would be aware of the issues that happened and were discussed. The DSB huddle is a time for nursing staff to prioritize the day's patient safety. Participants focus on a clear set of objectives, discussing safety events since the last huddle, and anticipate those prior to the next huddle.

\subsection{Data and Statistical Analysis}

In this study, AE (also known as accidents or harmful events) were defined as unintended harm to the patient by an act of commission or omission rather than by the underlying disease or condition of the patient [12]. NM (also called close misses or potential adverse events) are a type of potential hazard that did not reach the patient because of processes already in place that protected the patient from potential harm or by chance alone $[3,13]$.

The data of the electronic AE report system and the written huddle notes were analysed. Content analysis enabled classification of large amounts of textual data. The AE categories used in the study were based on 14 basic nursing quality indexes of clinical nursing in Guangdong Province, China. NM categories were based on the NM data reported and the literature. Statistical analyses were completed using SPSS 13.0.

\section{Results}

\subsection{Reporting Frequency and Rates of AE and NM}

Reporting frequency and rate of $\mathrm{AE}$ and NE are shown in Table 1. The results showed that implementing DSB huddles significantly increased the reporting rates of $\mathrm{AE}$ and $\mathrm{NM}(\mathrm{P}<$ $0.05)$.

Table 1. Reporting frequency and rate of $A E$ and $N M$.

\begin{tabular}{llll}
\hline & Inpatients (n) & AE, n (\%) & NM, n (\%) \\
\hline Pre-DSB & 1851 & $17(0.9 \%)$ & $10(0.5 \%)$ \\
Post-DSB & 1931 & $34(1.8 \%)$ & $138(7.1 \%)$ \\
$\chi^{2}$ & & 5.040 & 109.694 \\
P & & 0.025 & 0.000 \\
\hline
\end{tabular}

\subsection{Reporting Types of Safety Issues (Including AE and NM)}

Regarding safety issues including AE and NM, there were 27 safety issues pre-DSB, whereas post-DSB there were 172 . The reporting types are shown in Table 2 . Results showed that DSB huddles increased the self-reported rate of safety issues from $44.4 \%$ to $73.8 \%(\mathrm{P}<0.05)$ (Table 1$)$. Frequency of reporting types is shown in Figure 1.
Table 2. Reporting types of safety issues.

\begin{tabular}{lllll}
\hline & Pre-DSB & Post-DSB & $x^{2}$ & $\boldsymbol{P}$ \\
\hline Self-report & $12(44.4 \%)$ & $127(73.8 \%)$ & & \\
Witnessed report & $15(55.6 \%)$ & $45(26.2 \%)$ & 9.573 & 0.002 \\
Total & 27 & 172 & & \\
\hline
\end{tabular}

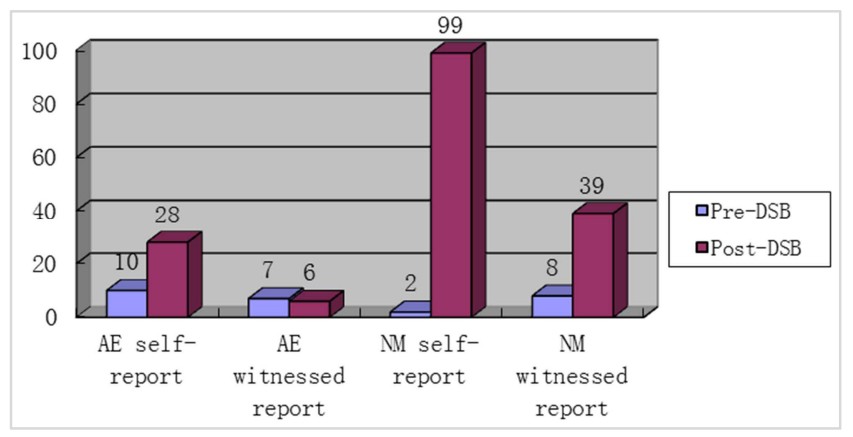

Figure 1. Frequency of reporting types.

\subsection{Categories of $A E$ and $N M$}

Pre-DSB huddles, AE reported by nurses were limited to medication errors, pressure sores, falls, and unplanned extubations (see Table 3). Medication errors were the most common adverse event, both before and after DSB huddles.

During the DSB huddles, nurses reported more dimensions of NM (see Table 4). After implementation of DSB huddles, NM reporting increased from 6 to 15 dimensions; the specific types and frequency are listed in Table 4 . The most common NM were those related to medication administration.

Table 3. Types and frequency of $A E$.

\begin{tabular}{lll}
\hline AE type & Pre-DSB n $=\mathbf{1 7}$ & Post-DSB n = 34 \\
\hline Medication error & $7(41.2 \%)$ & $13(38.2 \%)$ \\
Pressure sores & $4(23.5 \%)$ & $4(11.8 \%)$ \\
Falls & $1(5.9 \%)$ & $5(14.7 \%)$ \\
Unplanned extubation & $5(29.4 \%)$ & $11(32.4 \%)$ \\
Sharp-force injury & & $1(2.9 \%)$ \\
\hline
\end{tabular}

Table 4. Types and frequency of NM.

\begin{tabular}{lll}
\hline NM type & Pre-DSB & Post-DSB \\
\hline Medication administration & 3 & 38 \\
Transcription error & 2 & 3 \\
Noncompliance with policy/procedure & & 7 \\
Patient identification & 1 & 5 \\
Incorrect physician orders & & 6 \\
Incorrect procedure/process & & 17 \\
Lack of communication among the care & & 6 \\
team or department team or to physician & & 6 \\
Supplies/equipment & 2 & 15 \\
Patient monitoring & & 4 \\
Verification against original physician & & 4 \\
order & 1 & 3 \\
Mislabelled /missed specimen & & 10 \\
Staff communication with & & 8 \\
patient/family & & 6 \\
Medical Documents & 1 & 138 \\
Preoperative preparation & & \\
Unable to classify & 10 & \\
Total & & \\
\hline
\end{tabular}




\section{Discussion}

Incident reporting offers a valuable source of information regarding safety issues, but NM and AE remain underreported [14]. Many factors, such as perceived difficulty in using the reporting system [15], lack of training in use of the incident reporting process [16], time required to report errors [8], and culture, management behaviour, and professional barriers, such as fear, accountability, characteristics of nurses [17], can lead to underreporting of safety issues. Reporting of medication errors by nurses occurs in only $37.4 \%-67 \%$ of cases, which is challenging, as underreporting makes it impossible to analyse the process underlying all medication errors and NM. Several studies have estimated that the rate of medical errors far exceeds those disclosed by voluntary hospital reporting systems, and few studies have estimated the rate of NM [6]. Experts call for greater attention to $\mathrm{AE}$ in the medical field [18, 19], because NM occur several times before an adverse event, and many preventable deaths have a history of preceding related near-misses [20].

Previous studies identified medication administration (19\%) and transcription errors $(10 \%)$ as the most frequent types of NM [21]. Although used almost the same classification of NM, results showed some differences, after DSB huddles the top category of NM was still related to medication administration, but NM related to incorrect procedure/process and patient monitoring were the second and third reported by nurses. Experts estimate that NM occur 3-300 times more often than AE in health care settings [19], this study supports that too. After DSB implementation, NM occurred about four times as often as $\mathrm{AE}, \mathrm{AE}$ were 34 and $\mathrm{NM}$ were 138. The most common AE were medication error, occurred 13 times while NM occurred 38 times. NM events, in which harm is averted because of chance or intervention, typically precede a related AE [20], so must pay more attention to the NM that occurred in this study, otherwise they could lead to related AE. Improving the successful reporting of $\mathrm{NM}$ and $\mathrm{AE}$ requires a culture change at the institutional level [20, 22]. DSB provides a fast, open, transparent, and easy way for nurses to report, so it is easy for nurses to accept and they are willing to report $\mathrm{AE}$ and NM.

The study revealed that implementing DSB huddles increased the reporting rate of $\mathrm{AE}$ and $\mathrm{NE}$; the nurse self-reported rate of safety issues increased from $44.4 \%$ to $73.8 \%$. During the DSB huddles, safety issues were communicated verbally during the DSB huddles, which provided a less burdensome and more conversational mechanism by which to discuss sensitive issues. Additionally, the DSB huddles programme run by the unit's nurse manager at the scene incentivises nurses to report NM safety events, encourages improved communication and collaboration among team members, and possibly encourages staff members to report safety issues and provide timely and effective feedback to the nurse regarding the consequences of their actions. This increase team members' situational awareness about patient safety, facilitating identification of concerns and development of plans to mitigate these concerns, so nurses become more aware that reporting of all $\mathrm{AE}$ as well as NM is needed. Reporting of NM is vital to understanding individual and systemic problems that may lead to AE, and has the potential to provide information necessary to prevent future AE [14]. However, when they are not reported, the opportunity to learn from NM is lost. Implementing DSB helps to develop a culture of safety and awareness among all nurses. Furthermore, encouraging individuals to report events and timely analysis of events with feedback to the individual have been proposed to improve effective reporting of NM and AE. This study found a much higher frequency of NM reporting during DSB huddles; before implementation of DSB huddles, only $10 \mathrm{NM}$ were reported, while 138 were reported implementation of DSB huddles, 10 times more than before, and reporting of dimensions of NM increased from 6 to 15 dimensions. This shows that a majority of NM went unreported before implementation of DSB huddles, and nurses were more willing to share all the noteworthy patient safety issues within DSB huddles. As was evident in the DSB, nurses were more likely to report NM that did not cause patient harm, such as equipment problems and knowledge/attitude, and policies/process problems than individuals using the electronic reporting system. Although these events did not lead to patient harm, they had the potential to do so. NM that continue to occur will lead to AE.

\section{Conclusions}

In conclusion, $\mathrm{AE}$ and $\mathrm{NM}$ especially were vastly underreported before implementing DSB. It has more recently been considered that focusing on NM is more important in the medical field than focusing on AE. The reporting and analysis of NM may provide more information than relying on $\mathrm{AE}$ reporting alone [23]. DSB huddles increased the reporting rates of $\mathrm{AE}$ and $\mathrm{NM}$, improved the reporting dimensions of NM, and increased team members' situational awareness about patient safety. DSBs are usually employed hospital-wide, and few studies have studied DSB huddles conducted in the unit and their impact on the reporting of adverse events and NM. In this study, implementing DSB huddles in a unit is a feasible, effective strategy to improve and increase reporting of $\mathrm{AE}$, and of NM particularly. The findings can contribute to better management of patient safety and prevention of adverse incidents. DSB huddles conducted within a unit seem to provide a fast, open, transparent, and easy way for nurses to report $\mathrm{AE}$ and $\mathrm{NM}$, overcome time-consuming barriers, and so increase the effective reporting of $\mathrm{AE}$ and $\mathrm{NM}$, thus improving patient safety.

\section{References}

[1] Dimova R, Stoyanova R, Doykov I. Mixed-methods study of reported clinical cases of undesirable events, medical errors, and near misses in health care. Journal of evaluation in clinical practice. 2018; 24(4):752-757. 
[2] Clancy CM. Common formats allow uniform collection and reporting of patient safety data by patient safety organizations. American Journal of Medical Quality. 2010; 25(1):73-75.

[3] Chamberlain CJ, Koniaris LG, Wu AW, Pawlik TM. Disclosure of "nonharmful" medical errors and other events: duty to disclose. Arch Surg. 2012; 147(3):282-286.

[4] CJ C, LG K, AW W, TM P. Disclosure of "nonharmful" medical errors and other events: duty to disclose. Archives of Surgery. 2012; 147(3):282-286.

[5] Smith KS, Harris KM, Potters L, et al. Physician attitudes and practices related to voluntary error and near-miss reporting. Journal of Oncology Practice. 2014; 10(5): e350.

[6] Classen DC, Resar R, Griffin F, et al. 'Global trigger tool' shows that adverse events in hospitals may be ten times greater than previously measured. Health Aff. 2011; 30(4):581-589.

[7] Yoon RS, Alaia MJ, Hutzler LH, Iii JAB. Using "Near Misses" Analysis to Prevent Wrong-Site Surgery. Journal for Healthcare Quality. 2013; 37(2):126.

[8] Menon S, Singh H, Giardina TD, et al. Safety huddles to proactively identify and address electronic health record safety. Journal of the American Medical Informatics Association. 2016: ocw153.

[9] Goldenhar LM, Brady PW, Sutcliffe KM, Muething SE. Huddling for high reliability and situation awareness. Bmj Quality \& Safety. 2013; 22(11):899-906.

[10] Sediva I, Snelling L. Daily Safety Briefs (DSB) focus on improving safety at Hasbro Children's Hospital. R I Med J. 2018; 101(2):23.

[11] Hatva E. Daily Briefing Promotes Hospital-Wide Transparency And Patient Safety. Biomedical Instrumentation \& Technology. 2013; 47(6):489-492.

[12] Maurette P. [To err is human: building a safer health system]. (0750-7658 (Print)).

[13] Yu KH, Nation RL, Dooley MJ. Multiplicity of medication safety terms, definitions and functional meanings: when is enough enough? Quality \& Safety in Health Care. 2005; 14(5):358-363.
[14] Hamilton EC, Pham DH, Minzenmayer AN, et al. Are we missing the near misses in the OR? - underreporting of safety incidents in pediatric surgery. Journal of Surgical Research. 2018; 221:336-342.

[15] Meeks DW, Smith MW, Taylor L, Sittig DF, Scott JM, Singh H. An analysis of electronic health record-related patient safety concerns. Journal of the American Medical Informatics Association Jamia. 2014; 21(6):1053-1059.

[16] Ashcroft DM, Morecroft C, Parker D, Noyce PR. Likelihood of reporting adverse events in community pharmacy: an experimental study. Quality \& Safety in Health Care. 2006; 15(1):48.

[17] Vrbnjak D, Denieffe S, O'Gorman C, Pajnkihar M. Barriers to reporting medication errors and near misses among nurses: $\mathrm{A}$ systematic review. International journal of nursing studies. 2016; 63:162-178.

[18] Shojania KG, Wald H, Gross R. Understanding medical error and improving patient safety in the inpatient setting. Medical Clinics of North America. 2002; 8 6(4):847-867.

[19] Barach P, ., Small SD. Reporting and preventing medical mishaps: lessons from non-medical near miss reporting systems. Bmj. 2000; 320(7237):759-763.

[20] Van Spall H, Kassam A, Tollefson TT. Near-misses are an opportunity to improve patient safety. Current opinion in otolaryngology \& head and neck surgery. 2015; 23(4):292-296.

[21] Speroni KG, Fisher J, Dennis M, Daniel M. What causes near-misses and how are they mitigated? Plastic surgical nursing : official journal of the American Society of Plastic and Reconstructive Surgical Nurses. Jul-Sep 2014; 34(3):114-119.

[22] Larizgoitia I, Bouesseau MC, Kelley E. WHO Efforts to Promote Reporting of Adverse Events and Global Learning. Journal of Public Health Research,2,3(2013-12-01). 2013; 2(3): e29.

[23] Grant MJ, Larsen GY. Effect of an anonymous reporting system on near-miss and harmful medical error reporting in a pediatric intensive care unit. Journal of Nursing Care Quality. 2007; 22(3):213-221. 